\title{
APORTES A LA APROPIACIÓN SOCIAL DE TIC DESDE CONSEJOS COMUNITARIOS afrocolombianos de Corinto, Cauca
}

Walter Julián Quinchoa-Cajas ${ }^{*}$

\section{Resumen}

La apropiación social de TIC ha alcanzado mayor campo de análisis e investigación. Hace unos años, se concebía sin la complejidad que varios autores, organizaciones e instituciones le han venido reconociendo. Esto hace pensar que, a pesar de los múltiples esfuerzos realizados desde varios sectores, el uso y la apropiación aún siguen sin ser comprendidos profundamente en sus entornos locales. Se presenta una estrategia metodológica construida con organizaciones rurales para la apropiación social de las Tic a través de análisis etnográficos de los "entornos prácticos" en la interrelación sujetos-tecnologías, atendiendo sus dimensiones a partir de una investigación en Corinto, Cauca.

Palabras clave: antropología; apropiación social; estrategia; TIC; organizaciones.

\section{CONTRIBUTIONS TO SOCIAL APPROPRIATION OF ICT IN AFROCOLOMBIAN COMMUNITY COUNCILS FROM CORINTO, CAUCA}

\section{Abstract}

The social appropriation of ICT has gained a big space in the field of analysis and research. A few years ago, it was conceived without the complexity that several authors, organizations and institutions have recognized. This suggest that, despite the amount of efforts made by several sectors, the use and ownership are still not deeply rooted in their local environments. For this study we present a methodological strategy constructed with rural organizations for the social appropriation of ICT's through the analysis of the "practical environments" in the interrelationship between subjectstechnologies, attending their dimensions from an investigation in Corinto Cauca.

Magíster en Gestión de Organizaciones y Proyectos. Investigador del Grupo de Estudios Sociales Comparativos (GESC), Universidad del Cauca (Colombia). [jquinchoa@unicauca.edu.co], [https://orcid.org/0000-0001-5408-2759]. Recibido: 10 de septiembre de 2019 / Modificado: 6 de diciembre de 2019 / Aceptado: 6 de diciembre de 2019

Para citar este artículo

Quinchoa-Cajas, W. J. (2020). Aportes a la apropiación social de Tic desde consejos comunitarios afrocolombianos de Corinto, Cauca. opera, 27, 45-62.

Dor: https://doi.org/10.18601/16578651.n27.03 
Key words: Anthropology; social appropriation; strategy, ICT; organizations.

\section{INTRODUCCIÓN}

El presente artículo describe el resultado principal del proyecto de investigación sobre la apropiación social de tecnologías de la información y las comunicaciones (TIC) en dos consejos comunitarios afrocolombianos del departamento del Cauca. Las inquietudes surgieron a partir de experiencias previas a través de proyectos desarrollados con el Centro de Desarrollo Tecnológico CREATIC y el Grupo de Estudios Sociales Comparativos (GESC) de la Universidad del Cauca, que llevaron a plantear interrogantes sobre el fenómeno de la apropiación social. Estos cuestionamientos fueron el cómo, el porqué y el para qué se presenta la apropiación social de TIC en dos organizaciones rurales del Cauca, además de preocuparnos por el tipo de implicaciones que puede tener este proceso de apropiación en dichas organizaciones.

Para abordar estos interrogantes, en primer lugar, se acude a la estrategia analítica de relacionar cultura, sociedad y tecnología, y las modalidades de TiC con las que trabajan los consejos comunitarios, tales como computadores y sus programas e internet. Echeverría ha argüido que el desarrollo de las capacidades TIC "no solo ha de producirse a nivel individual, también tiene una faceta institucional y colectiva y la apropiación social requiere de una apropiación cultural" (2008, p. 173). Se trata de renovadas miradas de estas tecnologías dado que los procesos de cambios sociales en los cuales están inmersas hacen necesario reflexionar sobre aquellas definiciones en las que anteriormente fue más pertinente hablar de su aspecto técnico. Se busca que la estrategia metodológica contribuya a llenar el vacío que existe en las organizaciones rurales dado que se tiene apenas estimado el uso de las Tic. Por esta razón es que este esfuerzo de muchos actores sociales, quienes coadyuvaron en su construcción, es apenas un punto de partida en todo el proceso de apropiación social.

El artículo está estructurado en cuatro partes. La primera, arroja algunos elementos metodológicos de la investigación; la segunda, reflexiona sobre el concepto de organización y el papel de las tecnologías desde una aproximación etnográfica; la tercera, aborda el concepto de apropiación social de las TIC en el entorno colombiano, articulado a algunos antecedentes de apropiación social en el departamento del Cauca; por último, se presenta la estrategia que se generó tras la ejecución del trabajo de investigación realizado con la participación de los integrantes de los consejos comunitarios.

\section{ASPECTOS METODOLÓGICOS}

La estrategia empleada a lo largo de la investigación es la denominada triangulación metodológica (Cisterna, 2005). Después de acudir a las reuniones con los tres consejos comunitarios, dos de ellos aceptaron la realización del trabajo de campo antropológico a través de una apertura de ciclos de formación que se llevarían a cabo de acuerdo con su tiempo disponible, 
que sería los viernes y sábados de cada semana entre julio y diciembre de 2017.

Metodológicamente, se acordó establecer conversaciones sobre el tema y abrir un espacio de formación básico sobre Tic en los Kioscos Vive Digital ubicados en la Institución Educativa Núcleo Técnico Agropecuario con sede en la vereda La María, corregimiento El Jagual, y la sede educativa El Barranco del corregimiento que lleva su mismo nombre. Estos aspectos se denominan entornos prácticos basados en la "estrategia formativa" de ciclos de formación (Bermúdez, 2013). Al respecto Toboso (2013, p. 202), en su investigación sobre apropiación de tecnologías, ha planteado que:

En términos generales podríamos decir que los entornos prácticos de un grupo social dado son todos los espacios en los que el grupo sitúa sus discursos, entendiendo por tales discursos el entrelazamiento de prácticas, representaciones y valores compartidos por el grupo acerca del "objeto" particular de que se trate (en el caso que aquí nos ocupa, una tecnología determinada).

En estos entornos prácticos el investigador asumió, no solo el rol de observador $\leftrightarrow$ participante, sino el de facilitador del ciclo de formación como estrategia formativa con cada grupo de los consejos comunitarios; esta fue otra técnica aplicada que Geilfus (2002) denomina "grupos enfocados". En estos espacios participaron hombres y mujeres entre los 14 y 64 años de edad, cada uno con diferentes roles dentro de sus organizaciones, pero con la voluntad de querer aprender más sobre las TIC. Cada grupo estuvo conformado por cinco personas en el consejo comunitario El Barranco y doce en el consejo comunitario Jagual La
María. En cada sesión de trabajo se tomaron notas condensadas producto de las observaciones y la participación en las sesiones para luego convertirlas en notas expansivas a fin de ampliar los aspectos registrados. Cada ciclo de formación tuvo una guía metodológica que se construyó con cada grupo denominada "Formación en TIC desde consejos comunitarios". Estos entornos prácticos fueron estudiados a partir de una perspectiva etnográfica, esto es, se analizan cualitativamente sus características alrededor de las preguntas de investigación. La etnografía "en tanto metodología, [...] no sería tanto la técnica de investigación de la observación participante como la manera de abordar la investigación misma” (Restrepo, 2016, p. 32). En una libreta de campo se tomaron notas condensadas de lo observado y analizado para luego ser expandido (notas expansivas) en el trabajo de escritorio, donde se elabora y estructura el material obtenido (grabaciones, fotos, escritos).

Las organizaciones rurales estudiadas no tienen oficinas sofisticadas que cuenten con equipos de alta capacidad y estén a la vanguardia de las tecnologías. Por el contrario, los sitios identificados que proveen TIC son los denominados Kioscos Vive Digital "para la gente". Programa del Gobierno colombiano que se ha venido implementando en ámbitos rurales desde el año 2010 y que en este caso se localizan en sedes educativas rurales.

Para el desarrollo de la investigación fue relevante la apertura de los dos espacios de formación porque permitió asumir el rol de facilitador para emprender el ciclo de formación. La selección de las personas que hicieron parte del ciclo de formación quedó como 
responsabilidad de los representantes de cada consejo comunitario, se pidió compromiso en la participación dado que se esperaba que fueran activos y que dichos conocimientos le sirvieran a la organización. Así fue acordado y, un mes más tarde, se empezó con la identificación de los temas que se impartirían en estos espacios más el diagnóstico rápido de las TIC encontradas en los entornos prácticos con el fin de establecer el plan curricular y la apropiación de las Tic, así como la organización de la cual hacen parte para que respondiera a sus necesidades, lo que permitió establecer consideraciones que fueron corroboradas bajo la triangulación metodológica (Cisterna, 2005).

También se aplicaron técnicas como conversaciones (Guber, 2001) para dialogar sobre los procesos de apropiación de TIC; esta técnica fue la más adecuada para este tipo de organización. Por lo general, se encontró a personas calladas que paulatinamente fueron permitiendo el acceso al diálogo (estructuradamente) sobre este tema y su organización. En este sentido, los entornos prácticos fueron escenas culturales que permitieron analizar la interrelación entre los participantes y las modalidades de TIC como computadores, portátiles e internet. Dichas conversaciones individuales y colectivas estuvieron basadas en preguntas orientadoras que, poco a poco, permitieron ampliar y precisar información obtenida en la revisión secundaria de textos, que luego fue socializada con los participantes. El resultado de todo este proceso condujo a la construcción de una estrategia metodológica de apropiación de TIC que será descrita sucintamente en la cuarta parte de este artículo.

\section{ORGANIZACIÓN Y TECNOLOGÍA DESDE UNA VISIÓN ETNOGRÁFICA}

El punto de partida de la investigación y el acercamiento a los consejos comunitarios es comprender que este tipo de organización tiene una dinámica propia que, si bien hace parte de un sistema mucho más amplio como el económico y el político, no niega ser parte del sistema cultural en el cual están inmersas y que corresponde a ciertas lógicas propias de un sistema de pensamiento-lenguaje (Cruz, 2013, 2014; Chanlat, 2014). Así, cuando se emplea el concepto de organizaciones no se hace referencia a la ciencia de la administración, sino a la constitución de personas que son diversas dada su identidad constituida/construida con ese yo-Otro y con el "otro" a través de la historia (Larraín, 2017).

Existe un aspecto problemático en las posturas teóricas de la ciencia de la administración debido a su búsqueda de homogeneizar las prácticas organizacionales sin importar las particularidades de las personas. Estos discursos emergen en un sistema de pensamiento lógico-racional, donde lo que interesa es la racionalidad instrumental. Esta racionalidad significa que, en términos operativos y funcionales, la administración ortodoxa no tiene que pedir nada más para cumplir unos fines a partir de unos medios, es decir, que lo que importa es el resultado y no cómo se accede a él, lo que desdibuja la perspectiva ética que se debe tener al interactuar con actores sociales. En efecto, la administración ortodoxa, al quedarse en un enfoque técnico, plantea unos modelos para explicar cómo los medios se deben utilizar para el cumplimiento de los fines. En términos de 
Foucault (2008), los medios son dispositivos de función de la gestión, pero todo depende de la configuración que se les dé a los medios y fines (Rojas, 2003).

En este sentido las organizaciones, comprendidas como un fenómeno cultural (Morgan, 1991) -a diferencia de la perspectiva de las posturas históricas de la administración-, son autónomas y rompen con la idea de que tienen límites fijados y establecidos porque ellas se caracterizan por su dinámica a través del espacio-tiempo. Las teorías y los enfoques de la administración y de las organizaciones han carecido de orientaciones que reconozcan la condición humana de los sujetos que hacen parte de su entorno organizacional (Chanlat, 2014; Murillo, 2007). Montaño ha señalado que el estudio de las organizaciones tiene como característica la diversidad de perspectivas teóricas que las han abordado y pueden ser clasificadas en funcionales y analíticas. Estas últimas pueden ser divididas en dos: la operativa, que trata de reconocer las modalidades de estructuración y los principales procesos, y la crítica, que estudia los efectos de su acción tanto a nivel individual como social (Montaño, 2013).

Desde la antropología de las organizaciones, campo epistemológico emergente (Cely y Gómez, 2016), se han trazado dos focos de investigación: 1) el cuestionamiento de la conceptualización de cultura organizacional/ empresarial emergida de la administración; 2) un abordaje etnográfico de las organizaciones. Gonnet (2013) sostiene, siguiendo a $S$. Wright (1997), que la cultura no es algo que las organizaciones tienen, sino algo que estas son $y$, de esta manera, pueden problematizar- se en el sentido de que es posible y pertinente cuestionar propositivamente los procesos que desarrollan en los territorios locales.

Así pues, es necesario que las organizaciones se aborden comprendiendo otros puntos de vista, y no necesariamente desde perspectivas ortodoxas, sino de carácter explicativo y analítico, no interesadas por lo razonable productivo, sino que sean consecuentes con otras lógicas propias del lugar desde donde emergen. Por tanto, es preciso develarlas con el fin de comprenderlas. En este sentido, la apuesta final de los estudios organizacionales en América Latina no es constituirse en una disciplina como tal, "se trata a la vez de un esfuerzo organizacional, interorganizacional e internacional; aborda a la organización siempre en su relación con el individuo y la sociedad, el grupo y la comunidad" (Montaño, 2013). Es justamente esta perspectiva la que interesa para el abordaje de ambas organizaciones sujetos de estudio, esto es, los consejos comunitarios, porque reconoce la dinámica de las organizaciones y a los sujetos que hacen parte de estas. Los aspectos señalados son fundamentales ya que las organizaciones que se pretenden analizar están accediendo a apropiaciones sociales de tic, aspecto que sigue sin profundizar desde ellas mismas.

\section{Aproximación a las tecnologías desde dos perspectivas teóricas}

La relación entre cultura, sociedad y tecnología no es un tema nuevo en la antropología (Restrepo, 2016). Al respecto, M. Fisher (2007) ha demostrado ampliamente el acercamiento y la genealogía de las relaciones estrechas entre 
ciencia y tecnología a lo largo de la historia. A partir de la década de los noventa es notable el interés hacia dicho campo porque emergen las etnografías de la ciencia y la tecnología con el fin de hacer públicos los avances en estos temas, hasta constituirse no solo intereses investigativos, sino académicos y profesionales alrededor de esta temática (Escobar, 2005; Kreimer, 2007; Vacarezza, 1998).

Los antecedentes de los estudios de la tecnología datan de muchos siglos atrás (Fisher, 2007). Sin embargo, su abordaje epistemológico no ha sido siempre el mismo, a tal punto de plantear actualmente que las nuevas tecnologías deben estudiarse desde una perspectiva integral, a diferentes niveles $\mathrm{y}$ con un trato diferencial para Latinoamérica (Escobar, 2005).

En consonancia con los avances en este campo de estudio, se abordaron perspectivas teóricas como la propuesta por Lemonnier (1992), quien arguye que "la tecnología no es algo fuera de la cultura y la sociedad, sino una parte intrínseca de ella y 'las representaciones sociales de las tecnologías [son] el canal a través del cual los fenómenos sociales influyen en los sistemas tecnológicos'" (citado en Ellen, 1994, p. 652). Este autor entiende por representación social el "conjunto de ideas compartidas de un grupo social dado" (Lemonnier, 1992, p. 79). Por su parte, Tocancipá (2006) arguye que la tecnología es parte del entramado de la sociedad y la cultura donde esta se produce, es decir, tiene su propio locus y naturaleza. Empero, sus usos y lugar de apropiación no solamente se limitan a dichos contextos.

Interesa tomar en cuenta los aportes de Thomas Hughes, señala Pfaffenberger (1992), al argumentar la necesidad de buscar en el desarrollo de las nuevas tecnologías la trama de lo social, lo económico, lo legal, lo científico y lo político del contexto de la tecnología. Esto significa que una innovación tecnológica es exitosa en la medida en que trabajen articuladamente, lo social y lo tecnológico. Dicho lo anterior, la antropología de la tecnología es una de las perspectivas adecuadas para el abordaje de las organizaciones en tanto considera que la asimilación, el diseño y desarrollo de nuevas tecnologías se expresan en el contexto (Martínez y Arellano, 2004), tal como lo veremos en el caso de los consejos comunitarios y su apropiación social de las Tic.

Al comprender la amplitud y complejidad del concepto de tecnología, se abordan específicamente las tecnologías de la información y las comunicaciones (TIC) más allá de la conceptualización elaborada por el Ministerio de Tecnologías de la Información y las Comunicaciones de Colombia (Mintic) (Congreso de la República, 2009). Esta definición ha sido superada a partir de las investigaciones que se han realizado sobre apropiación social de tecnologías. En este sentido, es relevante acudir desde los estudios sociales de la ciencia y la tecnología a la conceptualización de tecnología que señala que "no solo son productos o procesos productivos, están propuestas desde una construcción y una percepción de las formas de organización social y productiva de un momento determinado y con necesidades específicas" (Thomas, 2010, citado en Escobar, 2014, p. 75). Al analizar este concepto, se infiere que esta tecnología enmarca un conjunto de operaciones de producción con normativas, sistemas legales, regulaciones comerciales que 
no se diferencia del artefacto tecnológico (Escobar, 2014).

Por tanto, estos hallazgos conducen a repensar y redefinir lo que entendemos por Tic. Escobar y Escobar (2013) seńalan que una vez entendidas las Tic de una forma convencional, tal como las define el Mintic, es necesario reflexionar en el desarrollo de un proceso de apropiación social de estas relacionado directamente con los usos y las prácticas que los actores tienen sobre aquellas y también con las habilidades y destrezas de los usuarios que ya tienen y que se adquieren al usarlas. Estos autores han planteado que, debido a las necesidades actuales del mundo, es indispensable una visión integral de las Tic. Otro planteamiento similar sobre estas tecnologías lo encontramos en los avances epistemológicos de Cobo (2009), quien seńala que lo más importante no es encontrar una única definición de TIC por cuanto existen muchas y diferentes, sino hallar aquellas que sean operacionales de acuerdo con el campo en cual se mueva la investigación. Argumento con el cual se está de acuerdo para abordar la investigación y, en particular, el fenómeno de la apropiación social desde los consejos comunitarios.

\section{El concepto de la apropiación social de tic en Colombia}

Recientemente, Escobar (2018) hace un llamado crítico frente a la falta de definición desde la política de ciencia y tecnología en Colombia sobre el término de apropiación social de la ciencia y la tecnología. Si bien se comparten algunos planteamientos de su artículo, también se cree necesario investigar desde las organiza- ciones de base para seguir realizando trabajo de campo y encontrar elementos comunes que coadyuven a la comprensión de los procesos locales iniciados en diferentes regiones en Colombia. No se desconocen los antecedentes de los inicios del empleo del concepto de apropiación social, sin embargo, es conveniente destacar que durante el año 2000 se da un giro epistemológico hacia el estudio del uso y la apropiación social de tecnologías incluidas las TIC, fundamentado en el apoyo institucional a través de los documentos del Consejo Nacional de Política Social y Económica (conpes) 3072 del año 2000 (Mintic, 2000) y 3457 de 2007 (Mintic, 2007).

Barón y Gómez (2012) argumentan que el CONPEs ha tenido dos fases en el avance de políticas de Tic en los últimos diez años en Colombia. La primera se dio entre los ańos 2002 a 2006 y su prioridad fue acceder a lo comunitario en servicios básicos de voz e internet, y entregar computadores a sedes educativas públicas. La segunda se estableció entre los ańos 2006 a 2010, cuyo fin fue fortalecer la provisión de acceso a banda ancha y los procesos de apropiación de las TIC en el ámbito educativo, específicamente en las instituciones públicas, con el fin de involucrar al sector productivo, en especial a micro, pequeñas y medianas empresas (Mipymes) y a las regiones para incentivar el uso y beneficio de las Tic.

El documento CONPES 3582 de 2009 establece la Política Nacional de Ciencia, Tecnología e Innovación, y define en un marco amplio en torno a la apropiación social, incluyendo el concepto innovación. Más allá de esto, la política diagnostica la baja apropiación de ciencia y tecnología a pesar de los programas y proyectos 
que se vienen implementando para incentivar la adquisición del conocimiento. En efecto, se formuló la Estrategia Nacional de Apropiación Social de la Ciencia, la Tecnología y la Innovación (ASCTI) (Colciencias, 2010), que es un insumo relevante para ser contextualizado a la luz de este tipo de investigaciones, las cuales pretenden caracterizar organizaciones étnicas como los consejos comunitarios.

Esta estrategia aporta lineamientos generales, por ello cabe destacar la existencia de antecedentes para un modelo de apropiación social de TIC en ámbitos rurales que solo se muestra en términos teóricos dado que no se evidencian en su propuesta aspectos prácticos para Latinoamérica como el de Gigli (2005), cuya iniciativa aporta a la comprensión de los factores de la apropiación de las tecnologías en lo rural. Desde otra perspectiva complementaria, Neüman arguye que "la apropiación social no es un concepto solamente sino también una categoría porque su complejidad como proceso en diferentes contextos así lo determina” (2008, p. 54).

Complementario a este concepto existe otro denominado resistencia cultural. Bonfil (2005) ha planteado que la apropiación es un segundo proceso de la resistencia cultural. También ha señalado que cuando una cultura toma decisiones propias frente a recursos propios es autónoma y frente a los ajenos es una cultura apropiada, en consecuencia, ambas conforman lo que se llama cultura propia (Bonfil, 1995). La resistencia, en consecuencia, no es el rechazo en sí mismo de las tecnologías, sino que "radica en el conocimiento y conciencia del sentido de la tecnología, en la excavación de los cimientos de la fachada tecnológica de la vida diaria" (Valderrama y Jiménez, 2005, p. 102).

Raigoso (2011) arguye que a partir del señalamiento de la variabilidad de la representación de apropiación no es necesario, ni posible ni conveniente "hallar" o establecer una definición precisa de este proceso y que es, entonces, más sugerente -para la política, las prácticas y para la comprensión de la relaciones entre la ciencia y la sociedad- un registro y análisis de las diferentes representaciones de apropiación, y un estudio de las condiciones y los contextos en que ellas se producen, se transforman y se validan (p. 206). La apropiación, concebida como un proceso propuesto de acciones intencionadas -como lo señala Barrio Alonso (citado en Colciencias, 2010)-, ha tomado diferentes representaciones (también denominadas significados, conceptos, nociones, tendencias, sentidos y definiciones) que varían de acuerdo con los agentes, intereses y contextos en que la representan (Raigoso, 2011).

Gonzalo (2014) ha venido abordando el tema conceptual de la apropiación social de Tic, clasificando las dimensiones de la apropiación para su comprensión (socioeconómica, praxiológica, subjetivo-individual, sociocultural, política y axiológica), y el enfoque discursivo que se le da para el uso y no uso de las tecnologías vinculado a la noción de apropiación tecnológica (Toboso, 2013).

En el siguiente apartado se describe el camino recorrido con los consejos comunitarios afrocolombianos, que constituye un aporte a aquella variabilidad de representaciones que toman las TIC en dichos entornos organizacionales. 


\section{HACIA LA CONSTRUCCIÓN DE UNA ESTRATEGIA METODOLÓGICA DE APROPIACIÓN SOCIAL DE TIC}

Los consejos comunitarios afrocolombianos son, de acuerdo con Bonfil (2005), una expresión de la resistencia cultural por cuanto esta organización trabaja arduamente para ser reconocida a nivel regional y nacional. Abordar el fenómeno de apropiación social de TIC con estas organizaciones étnicas y territoriales es dinámico y complejo porque parte de realidades desde lo rural (Siqueria y Osório, 2005), razón por la cual se propone que la estrategia metodológica debe tener de fondo un marco conceptual que la oriente y la sustente, si se quiere emprender el desafío del proceso del uso y apropiación de Tic.

Se entiende por estrategia un "conjunto de acciones planificadas sistemáticamente en el tiempo que se llevan a cabo para lograr un determinado fin. Su carácter participativo significa que lleva asociado un ejercicio de poder, de influencia y que se toma conciencia del mismo" (Aguado, 2014, p. 64). En este sentido, la estrategia fue pensada desde la organización que tiene un objetivo común según sus "'cosmologías' o representaciones generales acerca de la naturaleza de sus lazos sociales y su funcionamiento" ligadas a su cultura (Douglas, 1986, citado en Gonnet, 2014, p. 230) y sentidos de identidad. Bermúdez (2013) argumenta, para el caso de estrategias de apropiación comunitaria de TIC, que es importante tener una estructura para el diseño curricular, esto es, el plan de temas y un proceso educativo que tenga en cuenta la naturaleza de la población donde se lleve a cabo.
Un aspecto importante de reconocer son los diferentes niveles de apropiación identificados en los ciclos de formación que fueron analizados a la luz del planteamiento de Bermúdez (2013), quien señala tres niveles de apropiación: acceso equitativo, uso relevante y real apropiación. Alcanzar este último nivel es, sin duda, un desafío para aquellas organizaciones que decidan llevarlo a la práctica en tanto lo que se persigue es un alcance esperado de la estrategia que "se centra en la manera como la gente absorbe, sistematiza y transforma la información y las nuevas relaciones en nuevos conocimientos que pueden ser transmitidos a otros(as) y aplicados para resolver sus necesidades concretas" (p. 13). Se puede analizar el nivel en que se encuentra de apropiación con una etapa de análisis previo al momento de llevar a cabo la estrategia.

De otro lado, la investigación muestra que para implementar la estrategia metodológica se requiere de un actor social que lidere el proceso. Aquí acudimos a Geilfus (2002), quien ha planteado un nuevo enfoque del profesional que se acerca a poblaciones de ámbitos rurales, denominado "facilitador", a quien sugiere debe trabajar en tres aspectos: "uso de métodos apropiados, un cambio de actitudes, y el intercambio de informaciones entre todos los actores" (p. 7). Esto significa que el facilitador(a) siempre estará forjando un proceso dialógico al reconocer saberes locales de la organización (Freire, 2004).

La experiencia en trabajo de campo antropológico y los planteamientos de Bermúdez (2013) condujeron a reconocer la estrategia formativa como estrategia metodológica basada en un "modelo pedagógico de carácter 
formativo, no educativo, en tanto que asume como propósitos formar para la vida y el trabajo a partir del desarrollo de las competencias afectivas, cognitivas y expresivas del ser humano" (p. 5). La formulación o implementación de la estrategia no debe pasar por alto el entorno en el cual se desarrolla, esto es, debe reconocer nuevos enfoques sobre los cuales se trabaja el medio rural. Pérez (2005) argumenta que el medio rural es una entidad socioeconómica en un espaciogeográfico con cuatro componentes básicos: un territorio, una población, un conjunto de asentamientos y un conjunto de instituciones públicas y privadas.

En efecto, la estrategia metodológica que se propone surge como resultado de una investigación previa que se realizó durante el año 2017 en los corregimientos El Barranco y Jagual La María del municipio de Corinto, en el departamento del Cauca, cuyo desarrollo pasamos a presentar.

La primera acción estuvo orientada a la revisión de fuentes secundarias como el plan de desarrollo de los consejos comunitarios del norte del Cauca, plan de desarrollo departamental y municipal, investigaciones de universidades u observatorio étnico de la región, que fueron sistematizadas en una base de datos para su orden temático con el fin de facilitar la escritura de productos. Posteriormente, se realizaron los primeros acercamientos e identificación de organizaciones y actores sociales clave para la investigación, así como de los entornos prácticos entendidos como "el acercamiento de un grupo social a una tecnología mediada por formas de socialización tecnológica en diversos ámbitos de actividad" (Toboso, 2013, p. 202). Uno de los aspectos relevantes fue identificar los múltiples usos que una tecnología tiene en simultáneo de acuerdo con los entornos prácticos y también del no uso (Ferreira, Toboso y Pedraza, 2017).

La segunda acción estuvo encaminada a la elaboración, el ajuste y la socialización del proyecto con los diferentes actores sociales para recibir retroalimentación del mismo. Este proceso participativo fue fundamental en el tema de apropiación social de TIC.

La tercera acción tuvo como fin realizar el trabajo de campo etnográfico en dos organizaciones rurales de los denominados consejos comunitarios afrocolombianos del municipio de Corinto, tal como se ha venido mencionando. Después de acudir a las reuniones con ellos, dos aceptaron la realización del trabajo de campo antropológico a través de una apertura de ciclos de formación que se llevarían de acuerdo con su tiempo disponible entre julio y diciembre de 2017. Se acordó el establecimiento de conversaciones sobre el tema y abrir un espacio de formación básico (alfabetización digital) sobre Tic en los Kioscos Vive Digital, ubicados en la Institución Educativa Núcleo Técnico Agropecuario con sede en la vereda La María, corregimiento El Jagual, y en la sede educativa El Barranco, del corregimiento que lleva este mismo nombre. A estos espacios se les denomina entornos prácticos basados en la "estrategia formativa" de ciclos de formación (Bermúdez, 2013).

Como se mencionó, en estos entornos prácticos el investigador asumió el rol de facilitador del ciclo como estrategia formativa con cada grupo de los consejos comunitarios. A partir de la participación de las personas previamente escogidas para los entornos prácticos, 
se tomaron notas en la libreta de campo como registro de la experiencia. Esto permitió analizar el nivel de conocimiento y apropiación alrededor de las Tic y sobre la organización de la cual hacen parte, lo que permitió establecer consideraciones que fueron corroboradas bajo la triangulación metodológica (Cisterna, 2005). De la misma manera, se aplicaron técnicas como conversaciones (Guber, 2001) para dialogar en torno a los procesos de apropiación de TIC; aquí, como se mencionó, esta técnica fue la más adecuada para dialogar con las personas que conforman el consejo comunitario puesto que no generó timidez al interactuar con ellas.

La cuarta acción fue la elaboración de los reportes etnográficos como insumo para los productos de la investigación. Esta acción permitió una retroalimentación del proceso que se concibió como dialógico.

\section{RESULTADOS}

Como fruto del proceso analítico descrito se ha elaborado una propuesta de estrategia metodológica que se compone de seis momentos (figura 1). Cada momento cuenta con unos elementos prácticos que sirven de orientación para los actores sociales que la lleven a cabo. Es indispensable señalar que, dentro de la estrategia, la alfabetización digital es fundamental como punto de partida de acuerdo con el nivel de apropiación de TIC identificado. Para el ciclo de formación cada grupo debe tener un máximo de 12 personas, dado que es una cantidad adecuada desde lo pedagógico para establecer una estrecha relación entre el facilitador(a) y los participantes de la organización y, además, para clasificarlo por ciclos vitales. El facilitador(a) debe socializar el objetivo de la construcción colectiva de la estrategia metodológica con los consejos comunitarios afrocolombianos reconociendo las instancias clave para desarrollarla.

Cada momento de la estrategia es descrito brevemente a continuación. El momento 1 , identificación y caracterización de actores sociales, es sumamente importante dado el interés de reconocer con quiénes se empezará a desarrollar la estrategia de apropiación. Algunos autores plantean que esta actividad lleva alrededor de dos meses en trabajo de campo. En este sentido, es indispensable tener alguna clasificación de los actores sociales dado que no todos cumplen la misma función y rol en los territorios; algunas veces tienen competencias dentro de este y otras veces no la tienen. No obstante, esto hace parte del grado de conocimiento que se tenga sobre ellos. Una orientación en torno a los actores sociales es que pueden ser clasificados como actores públicos, locales e interesados, independientemente de la escala en la que trabajen, dado que pueden ser globales, nacionales, regionales y locales.

El momento 2, caracterización de las organizaciones, se basa en los insumos de la fase previa dado que es un referente para acercarse más a las organizaciones con las cuales se llevará a cabo la estrategia de apropiación social de las Tic. Aquí reiteramos que las organizaciones deben ser entendidas como cultura en tanto son dinámicas, responden a necesidades en contextos específicos y están constituidas por diversos actores sociales, entre los cuales se encuentran aquellos con quienes actúan en su quehacer cotidiano. Esto sugiere estudiar 


\section{FIGURA 1. ESTRUCTURA DE LA ESTRATEGIA METODOLÓGICA DE APROPIACIÓN SOCIAL DE TIC}

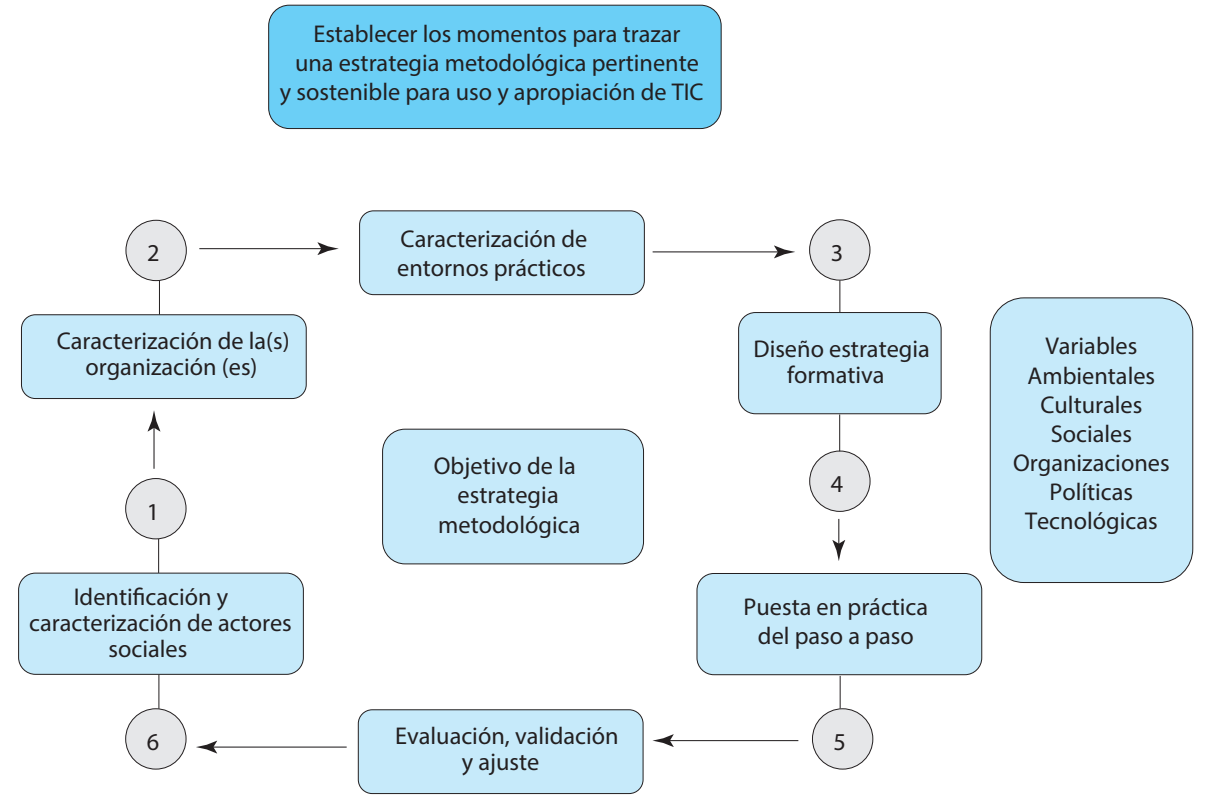

a las organizaciones más allá de sus lugares internos de trabajo dado que la particularidad de ellas es estar en contacto con diversidad de actores sociales. En este sentido, es necesario comprender su visión y misión, su proyección, sus temores, sus potencialidades, con el fin de enfocarse en las necesidades reales que tienen en los territorios donde se desenvuelven o desarrollan su trabajo o su vida misma (Echeverría, 2008). A las organizaciones no las podemos entender a través de medios o instrumentos que se plantean desde el exterior de ellas, al contrario, se deben entender al interior de las mismas en relación con estos factores y actores sociales quienes también hacen parte de su constitución, de su ser.

El momento 3, caracterización de entornos prácticos, destaca la importancia de recocer las nociones de tiempo que existen en los entornos rurales. No es posible que las nociones de tiempo lineales sean aquellas que primen en la estrategia metodológica, por el contrario, la estrategia para promover el uso y la apropiación de las Tic debe reconocer que en ámbitos rurales existen otras formas de percibir y vivir el tiempo en tanto la dinámica es otra y no corresponde a las que se tienen en los entornos urbanos.

La importancia de conocer de primera mano los entornos prácticos se debe a que el facilitador(a) tiene la oportunidad de saber si existe o no conexión a internet, con qué tipo de equipos se cuenta, si existe o no energía eléctrica, etc. Estos aspectos del entorno, tanto interno como externo de la organización, hacen que tenga un conocimiento amplio de 
lo que esta "es", de lo que la compone, de lo que la constituye y de quienes hacen parte de la misma.

El momento 4, diseño de la estrategia formativa, señala que una vez obtenida esta información amplia y detallada de la organización, los actores sociales implicados deben proponer la estrategia formativa de cómo se llevará a cabo. La información de contexto y de caracterización de actores permitirá plantear la forma adecuada de trabajar en la estrategia que, en lo posible, debe contar con un enfoque pedagógico que posibilite su comprensión.

Las características de la estrategia formativa son: debe identificar el número de personas con las que deberá realizarse, las condiciones del entorno práctico, el objetivo de la formación, las temáticas por impartir, el tiempo requerido para ello (acordando horario), y establecer normas para el espacio de formación. Existen dos bloques sobre los cuales se puede basar la estrategia formativa en cuanto a su contenido, esto es, en alfabetización digital o en temas de interés de la organización. No necesariamente deben ser impartidos como temas separados, sino que incluso se pueden combinar al poner en práctica temas estudiados. Se deben promover programas libres, que tengan acceso abierto para las organizaciones dado que no es posible incentivar la compra de programas costosos si se es consciente de la naturaleza de las organizaciones con quienes se está trabajando. Para la contextualización de las TIC es oportuno preguntarse para qué y por qué usarlas desde su enfoque integral. Los temas que le dan contenido a la estrategia formativa deben estar alineados con los planes de etnodesarrollo o de vida. Esto es importante porque ya han existido ejercicios de levantamiento de necesidades y las TIC deberían entrar a apoyar su misión y visión.

Algunos autores plantean que esto puede llevarse a cabo presencial o virtualmente; sin embargo, esto dependerá de las condiciones de la conectividad que, en ámbitos rurales, muchas veces tiene baja capacidad para soportar la conexión a internet. Se recuerda que aquí el facilitador(a) juega un rol clave porque debe estar al tanto de lo que requieran los actores locales para su desempeño. Se recomienda estar en contacto con los participantes para dinamizar los grupos de trabajo. El diseño de la alfabetización digital se basa en las siguientes preguntas: ¿¿cuáles son sus necesidades? ¿Qué niveles de formación tiene la población? ¿Cuál es el contexto educativo de la población? ¿Qué grupos de edad conforman a la población? ¿Cuántos son? (Bermúdez, 2013).

El momento 5, puesta en práctica de la estrategia metodológica, exige que el facilitador(a) tenga conocimientos técnicos básicos y liderazgo para acompañar el proceso. Entre las características y el perfil de esta persona se destacan: debe pertenecer a la organización, con vocación de trabajar y con alto compromiso para con esta. Una de las cualidades que debe tener el facilitador o liderazgo que encabeza el ciclo de formación es que debe sostener una comunicación fluida con sus participantes dado que a través de este diálogo abierto y sincero se llega a una confianza que permite tener un espacio tranquilo de aprendizaje. Es importante que la organización crea relevante la sistematización de la elaboración de todo el proceso de la estrategia. 
Argüimos que al hacer esto, se están generando elementos que se vuelven referentes para darle sostenibilidad al proceso y la organización empieza a contar con materiales propios para su consulta en aras de fortalecerse.

La finalización de la puesta en marcha de la estrategia puede hacerse con una evaluación que convoque a una actividad colectiva; en los consejos comunitarios lo denominan "compartir", lo cual permite, si es del caso, entregar los certificados o agradecer la participación de la organización. Esto genera cada vez más confianza entre los actores sociales involucrados en dicho proceso. La experiencia en trabajo de campo antropológico en el mes de diciembre fue hacer parte del "compartir" organizado por el consejo comunitario El Jagual La María, donde fue preciso entablar una conversación más abierta e informal, a fin de reconocerse cada uno como participante.

\section{CONSIDERACIONES FINALES}

La trayectoria histórica de los consejos comunitarios afrocolombianos en el municipio de Corinto muestra que aún existe la necesidad de seguir aportando desde la antropología de las organizaciones y demás posturas teóricas y metodológicas, al fortalecimiento de la reflexión y el análisis del fenómeno de apropiación social de TIC. Sin embargo, el camino recorrido para trazar la estrategia metodológica a través de su participación hace notar la posibilidad de seguir indagando desde su tejido social interno. La investigación realizada participativamente apunta a seguir fortaleciendo estrategias formativas para tomar ventaja de la infraestructura tecnológica existente en los espacios logrados a través de su gestión social. El reto está en tomar como guía las diferentes etapas de la estrategia para paulatinamente darle contenido de acuerdo a la naturaleza de cada consejo comunitario, pues cada uno tiene unas características particulares, y para hacer valer el derecho al acceso y uso de las Tic dado que la apropiación social de estas se manifiesta en la vida práctica (Echeverría, 2008).

Esta propuesta reconoce a los participantes como actores claves del desarrollo de la estrategia y no como usuarios pasivos de un modelo. Esta investigación logró repensar las Tic desde una visión crítica en aras de afianzar su uso al interior de las organizaciones étnicas de acuerdo con su Plan de Etnodesarrollo (ACONC, 2015-2035). La propuesta articula dos visiones aparentemente distantes, pero claves para el desarrollo de procesos de apropiación. Por una parte, el "objetivo de la estrategia metodológica” que permite a agencias estatales, organizaciones interfaz o actores del Gobierno central o regional proponer sus programas. De otra parte, el "establecimiento de los momentos para trazar una estrategia metodológica pertinente y sostenible para el uso y apropiación de TIC" permite que los actores sociales y participantes del ejercicio planteen sus expectativas frente a los programas antes propuestos. La conciliación entre estos dos frentes no solo posibilita una mejor articulación con la política pública de apropiación de TIC, sino que permite un ejercicio mucho más efectivo en virtud de la orientación de las sesiones de trabajo hacia las particularidades que realmente interesan, afectan y conectan a los participantes. 
Abordar organizaciones rurales desde perspectivas que reconocen que ellas son cultura en tanto son dinámicas y complejas responde a unas necesidades en contextos específicos, constituidos por distintos actores sociales, y en relación con diversidad de organizaciones; sugiere estudiar a las organizaciones más allá de sus lugares internos de trabajo, para lo cual se acude a la antropología y a los estudios críticos acerca de estas.

Una de las reflexiones que suscita esta investigación es que el fenómeno de la apropiación social es complejo y dinámico, y que no se acaba solo en la formulación de la estrategia; por el contrario, la comprensión de la dinámica de las organizaciones y del mundo de vida en el cual están inmersas resulta cambiante a través del tiempo. El desafío está en emplearla como guía para que las organizaciones sociales de base cada vez más participen activamente de la gestión de procesos de TIC.

Esta propuesta abre posibilidades para continuar afianzando el fenómeno de apropiación social a partir del abordaje de una concepción integral de las TIC y las modalidades con las cuales trabajan las comunidades rurales. Es claro que existe un número considerable de aportes frente a este fenómeno (v. gr. Colnodo, et al., 2007), lo relevante es que en estos territorios ya se viene trabajando de la mano de otras instituciones gubernamentales y no gubernamentales para formar a líderes locales sobre TIC que pueden seguir liderando estas estrategias que respondan a necesidades de la región en las organizaciones locales. Se trata de renovadas miradas de estas tecnologías dado que los procesos de cambios sociales en los que están inmersas hacen necesario reflexionar sobre aquellas definiciones en las que anteriormente fue más pertinente hablar de su aspecto técnico, situación que ha cambiado a partir de un abordaje de la antropología social de la tecnología y de los estudios sociales de estas, los cuales están basados en aportes multidisciplinarios propios para el abordaje de fenómenos complejos de la realidad.

\section{REFERENCIAS}

Aguado, T. (2014). Estrategias participativas de intervención socioeducativa. En P. Mata (ed.), Propuestas de intervención desde un enfoque participativo (pp. 64-97). Madrid: Universidad nacional de Educación a Distancia.

Alonso, C. (2008). La apropiación social de la ciencia: nuevas formas. Revista CTS, 4(10), 213-225.

Asociación de Consejos Comunitarios del Norte del Cauca (aconc) (s. f.). Plan de Etnodesarrollo de las comunidades negras del norte del Cauca 2015-2035 ¡Cambios para vivir mejor! (documento inédito).

Barón, L. y Gómez, R. (2012). De la infraestructura a la apropiación social: panorama sobre las políticas de las Tecnologías (TIC) de la Información en Colombia. Signo y Pensamiento, (61), 38-55.

Bermúdez, J. (2013). Más allá del acceso. Estrategias y herramientas para promover la apropiación de Nuevas Tecnologías en procesos comunitarios. Bogotá: Fundación internacional de pedagogía conceptual Alberto Merani y Fundación Tecnológica San Francisco de Asís.

Bonfil Batalla, G. (1995). El etnodesarrollo: sus premisas jurídicas, políticas y de organización. En G. Bonfil Batalla. Obras escogidas (t. 2, pp. 464-480). México: INAH, INI. 
Bonfil Batalla, G. (2005). México profundo. Una civilización negada. México: Random House Mondadori.

Cely-López, C. y Gómez-Niño, O. (2016). Una perspectiva antropológica de las organizaciones desde la caracterización sociocultural, administración y contabilidad social. Caso institución de educación superior. Cuadernos de Contabilidad, 17(43), 183-201.

Chanlat J. F. (2014). The forgotten contributions of the French schools of anthropology to the foundations of anthropological perspectives in the Anglophone universe: A comment on Morey and Luthans. Journal of Organizational Ethnography, 3(1).

Cisterna, F. (2005). Categorización y triangulación como procesos de validación del conocimiento en investigación cualitativa. Theoria, 14(1), 61-71.

Cobo, R. (2009). El concepto de tecnologías de la información. Benchmarking sobre las definiciones de las TIC en la sociedad del conocimiento. Zer, 14(27), 295-318.

Colnodo, Universidad Autónoma de Occidente, UAO y Programa Compartel del Ministerio de Comunicaciones de Colombia Telecentre.org. (2007). Guía para fortalecer la apropiación social en Centros de Acceso Comunitario a Tecnologías de Información y Comunicación (TIC). Recuperado de https:// colnodo.apc.org/apc-aa-files/511 feaa28064b75 833d25a46c040d121/guia_para_fortalecer_la_ apropiacion_1.pdf

Congreso de la República (2009). Ley 1341 de 2009. Diario Oficial, 47, 1-53. Bogotá:

Consejo Nacional de Política Económica y Social (CONPES) (2009). CONPES 3582. Politica Nacional de Ciencia, Tecnología en Innovación. Bogotá: CONPES.

Cruz, F. (2013). Pensamiento, obsesión por la verdad y gestión: legitimidad de los estudios críticos orga- nizacionales. En Estudios críticos de la organización. Qué son y cuál es su utilidad (pp. 129-146). Cali: Universidad del Valle.

Cruz, F. (2014). Modernidad, sentimientos negativos y conflicto social en Colombia. Cuadernos de Administración, 19(29), 100-112.

Departamento Administrativo de Ciencia, Tecnología e Innovación (Colciencias) (2010). Estrategia Nacional de Apropiación Social de la Ciencia, la Tecnología y la Innovación (ASCTI). Recuperado de http://190.242.114.60/colcienciasdev/sites/ default/files/ckeditor_files/estrategianacionalascti.pdf

Echeverría, J. (2008). Apropiación social de las tecnologías de la información y la comunicación. Revista CTS, 10(4), 171-182.

Ellen, R. (1994). Reseńa. Elements for an Anthropology of Technology by Pierre Lemonnier. Technology and Culture, 35(3), 652-654.

Escobar, A. (2005). Bienvenidos a Cyberia: notas para una antropología de la cibercultura. Revista Estudios Sociales, (22), 15-35.

Escobar, J. y Escobar, D. (2013). Fortalecimiento de capacidades en el uso y apropiación social de las Tecnologias de la información y las comunicaciones (TIC): Aportes desde los telecentros. Cali: Universidad Autónoma de Occidente.

Escobar, J. (2014). ¿ ¿Sociedad de la Información? Miradas sobre la apropiación social de las tecnologías. Cali: Universidad Autónoma de Occidente (UAO).

Escobar, J. (2018). La apropiación social de la ciencia y la tecnología como eslogan: un análisis del caso colombiano. Revista CTS, 38(13), 29-57.

Ferreira, M., Toboso, M. y Pedraza, F. (2017). Metodología para el análisis de la accesibilidad tecnológica de las personas con discapacidad: triangulación y elaboración de indicadores. Cuadernos de Gobierno y Administración Pública, 4(1). 
Fisher, M. (2007). Four genealogies for a recombinant anthropology of science and technology. Cultural Anthropology, 22(4), 539-615.

Foucault, M. (2008). Tecnologias del yo y otros textos afines. Buenos Aires: Paidós.

Freire, P. (2004). Extensión o comunicación. La concientización en el medio rural. México: Siglo xxI Editores.

Geilfus, F. (2002). 80 herramientas para el desarrollo participativo. Diagnóstico, planificación, monitoreo y evaluación. San José: Instituto Interamericano de Cooperación para la Agricultura (IICA).

Gigli, J. (2005). Hacia un modelo de apropiación de las tecnologias de la información y la comunicación en ámbitos rurales. Recuperado de http://www. juangigli.com/wp-content/uploads/hacia_un_ modelo_de_apropiacion_de_tic_(O)en_ambitos_rurales.pdf

Gonnet, J. (2013). Cultura, organizaciones y antropología. Avá, 21, 151-170.

Gonnet, J. (2014). Cosmologías y orden social organizacional. Sociológica, 29(81), 227-260.

Gonzalo, D. (2014). Una aproximación conceptual a la "apropiación social" de TIC. Question, 1(43), 17-31.

Guber, R. (2001). La etnografia. Método, campo y reflexividad. Bogotá: Norma.

Kreimer, P. (2007). Estudios sociales de la ciencia y la tecnología en América Latina: ¿para qué? ¿Para quién? Redes, 13(26), 55-64. Recuperado de http://www.redalyc.org/articulo.oa?id=90702603

Larraín, J. (2017). Ideología e identidad. Cuadernos de Teoría Social, 3(6), 8-21. Recuperado de http:// www.cuadernosdeteoriasocial.udp.cl/index.php/ tsocial/article/view/48

Martínez, R. y Arellano, A. (2004). Reseña de "Nuevas Tecnologías y Cultura" de C. Bueno y M. J. Santos (coords.). Convergencia, 11(35), 375-380.
Ministerio de las Tecnologías de la Información y la Comunicación (2000). conpes 3072. "Agenda de Conectividad. Colombia”. Bogotá: Mintic.

Ministerio de las Tecnologías de la Información y la Comunicación (2007). conPes 3457. "Lineamientos de política para reformular el Programa Compartel de Telecomunicaciones Sociales". Recuperado de http://www.mintic.gov.co/portal/604/articles3503_documento.pdf

Montaño, L. (2013). Los estudios organizacionales. Revisando el papel de la critica. En Estudios críticos de la organización. Qué son y cuál es su utilidad (pp. 21-46). Cali: Universidad del Valle.

Morgan, G. (1991). Imágenes de las organizaciones. México: Alfaomega.

Murillo, G. (2007). Grandes paradigmas en el desarrollo del pensamiento administrativo. En Teorías clásicas de la organización y del management (parte I). Bogotá: Editorial Kimpres.

Neüman, M. (2008). La apropiación social como práctica de resistencia y negociación con la modernidad. Anuario Ininco / Investigaciones de la comunicación Caracas, 20(1), 46-78.

Pérez, E. (2005). Hacia una nueva visión de lo rural. Repensando el desarrollo rural. En N. Giarracca (comp.), Una nueva ruralidad en América Latina (parte I, pp. 17-31). Buenos Aires: CLACso.

Pfaffenberger, B. (1992). Social anthropology of technology. Annu Rev Anthropology, (21), 491-516.

Raigoso Camelo, C. E. (2011). Apropiación social de la ciencia, la tecnología y la innovación: variabilidad de la representación. En T. Pérez-Bustos y M. Lozano Borda (eds.), Ciencia, tecnología y democracia: reflexiones en torno a la apropiación social del conocimiento (pp. 205-212). Medellín: Universidad EAfIT- Colciencias. 
Restrepo, E. (2016). Escuelas clásicas del pensamiento antropológico. Cuzco: Impresiones Gráficas Meta Color.

Rojas, W. (2003). Modernidad \& Humanidad: lo inhumano en la organización y en el trabajo. Cali: Universidad del Valle.

Siqueria, D. y Osório, R. (2005). El concepto de lo rural. Repensando el desarrollo rural. En N. Giarracca (comp.). Una nueva ruralidad en América Latina (parte I, pp. 71-84). Buenos Aires: ClaCso.

Toboso Martín, M. (2013). Entre el uso y no uso de la tecnología: un enfoque discursivo de la apropiación tecnológica. Intersticios, 7(2), 201-214.

Tocancipá, J. (2006). Relaciones ciencia, sociedad y tecnología: algunas consideraciones entre conocimiento(s), uso(s) y apropiaciones) sociales. En Una construcción interdisciplinaria sobre el desarrollo tecnológico y su impacto en el entorno (pp. 20-40). Popayán: Universidad del Cauca.

Vacarezza, L. (1998). Ciencia, tecnología y sociedad: el estado de la cuestión en América Latina. Revista Iberoamericana de Educación, (18), 13-40.

Valderrama, A. y Jiménez, J. (2005). Tecnología, cultura y resistencia. Revista de Estudios Sociales, (22), 99-103.

Wright, S. (1997). Culture in anthropology and organizational studies. En S. Wright, (ed.), Anthropology of Organizations (pp. 1-31). London and New York: Routledge. 